\title{
EPIDEMIOLOGISCHE CHARAKTERISIERUNG VON FÄLLEN VON ANGEBORENER SYPHILIS IN NORDBRASILIEN, VON 2014 BIS 2019
}

\section{ORIGINALER ARTIKEL}

ALMEIDA, Carolina Gomes ${ }^{1}$, ÁVILA, Gabriel Pereira², TEIXEIRA, Isabelly Montenegro $^{3}$, RODRIGUES, Raíza Júlia Viana ${ }^{4}$, DIAS, Claudio Alberto Gellis de Mattos $^{5}$, OLIVEIRA, Euzébio de ${ }^{6}$, DENDASCK, Carla Viana ${ }^{7}$, ARAÚJO, Maria Helena Mendonça de ${ }^{8}$, FECURY, Amanda Alves ${ }^{9}$

\section{ALMEIDA, Carolina Gomes. Et al. Epidemiologische Charakterisierung von Fällen} von angeborener Syphilis in Nordbrasilien, von 2014 bis 2019. Revista Científica Multidisciplinar Núcleo do Conhecimento. Jahrgang 05, Ed. 12, Vol. 14, S. 20-31. Dezember 2020.

ISSN: 2448-0959, Zugangslink:

https://www.nucleodoconhecimento.com.br/gesundheit/nordbrasilien, DOI: 10.32749/nucleodoconhecimento.com.br/gesundheit/nordbrasilien

\section{ZUSAMMENFASSUNG}

Syphilis ist eine Krankheit, die durch das Bakterium Treponema pallidum verursacht wird, das zu einem großen Teil durch sexuelle Übertragung erworben wird.

\footnotetext{
${ }^{1}$ Akademiker des medizinischen Kurses der Föderalen Universität von Pará (UNIFAP).

${ }^{2}$ Akademiker des medizinischen Kurses der Föderalen Universität von Pará (UNIFAP).

${ }^{3}$ Akademiker des medizinischen Kurses der Föderalen Universität von Pará (UNIFAP).

${ }^{4}$ Akademiker des medizinischen Kurses der Föderalen Universität von Pará (UNIFAP).

${ }^{5} \mathrm{PhD}$ in Theorie- und Verhaltensforschung (UFPA). Professor und Forscher am Bundesinstitut von Amapá (IFAP), Macapá Campus, AP. Professor und Forscher im Graduate Program in Professional and Technological Education (ProfEPT), IFAP, Santana Campus.

${ }^{6} \mathrm{PhD}$ in Tropenkrankheiten (UFPA). Professor und Forscher an der Föderalen Universität Para (UFPA), Campus Castanhal, PA.

${ }^{7}$ Theologe, PhD in Clinical Psychoanalysis. Er arbeitet seit 15 Jahren mit Scientific Methodology (Research Method) in der wissenschaftlichen Produktionsberatung von Master- und Doktoranden. Der Spezialist für Marktforschung und -forschung konzentrierte sich auf Gesundheit. Doktorand in Kommunikation und Semiotik (PUC SP).

${ }^{8}$ Master in Lehre und Gesundheitswissenschaften. Professor und Forscher an der Föderalen Universität von Amapá (UNIFAP), Macapá Campus, AP.

${ }^{9} \mathrm{PhD}$ in Tropenkrankheiten (UFPA). Professor und Forscher an der Föderalen Universität von Amapá (UNIFAP), Macapá Campus. Professor und Forscher im Graduate Program in Health Sciences (UFPA). Prorektor für Forschung und Graduiertenstudien an der Föderalen Universität am Amapá (UNIFAP).

RC: 96427

Zugangslink: https://www.nucleodoconhecimento.com.br/gesundheit/nordbrasilien
} 
Angeborene Syphilis ist eine ansteckende Erkrankung der vertikalen Übertragung (von der Mutter auf den Fötus). Ziel dieser Studie war es, die Anzahl der gemeldeten Fälle von angeborener Syphilis im Norden Brasiliens zwischen 2014 und 2019 darzustellen und das epidemiologische Profil der Fälle zu charakterisieren. Deskriptive, querschnittliche und retrospektive Recherchen wurden mit Hilfe der DATASUSDatenbank durchgeführt. Im Ausgewertetzeitraum stieg die Zahl der Fälle, insbesondere der Bundesstaat Amazonas, der die meisten Meldungen vorlegte. In Bezug auf die Schwangerschaftsvorsorge erbringen Frauen durchschnittlich viermal mehr Leistungen als diejenigen, die dies nicht taten. Frauen mit mütterlicher Schulnote von der 5. bis zur 8. unvollständigen Grundschule hatten eine höhere Anzahl von Fällen der Krankheit. Obwohl die nördliche Region eine hohe pränatale Leistung aufwies, wurden die meisten Fälle von angeborener Syphilis erst nach der Entbindung diagnostiziert, was auf falsche Interpretationen in Bezug auf die Tests und daraus resultierende Fehler in Diagnose und Behandlung hindeutet. Da die frühe Form (Entstehung bis zum 2. Lebensjahr) die Mehrheit der Anzahl der Fälle ist, gibt es eine günstige Entwicklung der Krankheit.Schulierung und Einkommen scheinen Faktoren zu sein, die die späte Diagnose der Krankheit beeinflussen. Es besteht ein Bedarf an erhöhten behandelten Partnern, wodurch die Übertragung von Syphilis und folglich angeborener Syphilis reduziert wird.

Schlüsselwörter: Angeborene Syphilis, Nördliche Region, Pränatal.

\section{EINLEITUNG}

Syphilis ist eine systemische, chronische, heilbare und exklusive bakterielle Infektion des Menschen. Unbehandelt entwickelt es sich zu Stadien unterschiedlicher Schwere und kann Organe und Systeme des Körpers betreffen (BRASIL, 2018). Der Erreger der Syphilis ist das Bakterium Treponema pallidum, eine Spirochäte, die in den meisten Fällen beim Geschlechtsverkehr erworben wird. Die Krankheit wird nach ihren verschiedenen Übertragungswegen in erworbene Syphilis und angeborene Syphilis eingeteilt (CAVALCANTE et al., 2012, ROCHA et al., 2020). 
Angeborene Syphilis ist das Ergebnis einer hämatogenen Ausbreitung von T. Pallidum , der infizierten schwangeren Frau, die wegen ihres Konzeptus unbehandelt oder unzureichend behandelt wird, transplazent (CRT, 2016). Die Übertragung kann während der gesamten Schwangerschaft auftreten, und das Risiko ist bei schwangeren Frauen mit primärer oder sekundärer Syphilis (GUINSBURG; SANTOS, 2010, BARROS et al., 2020).

Es ist eine Krankheit, die für etwa $40 \%$ der perinatalen Mortalitätsraten, $25 \%$ der Totgeburten, 14\% der neonatalen Todesfälle verantwortlich ist (CARDOSO et al., 2018). Darüber hinaus kann es schwerwiegende Folgen für den Conceptus als systemische Komplikationen nach sich ziehen, sei es bei früher angeborener Syphilis (Knochen-, neurologische, hämatologische und hepatosplenische Beteiligung) oder spät (Stigmata, die sich aus Umbau und Knochendeformitäten ergeben) (MOTTA et al., 2018).

Die wirksame Bekämpfung der Syphilis hat als grundlegende Prämisse ein serologisches Screening und die angemessene Behandlung von Schwangeren und Sexualpartnern, da die Qualität der Schwangerschaftsvorsorge und der Geburt eine wichtige Determinante für die Verringerung der vertikalen Übertragung ist. Penicillin ist das Medikament der Wahl bei der Behandlung von Syphilis und das einzige, das für schwangere Frauen indiziert ist: Es hat eine Wirksamkeit von 98\% bei der Vorbeugung von angeborener Syphilis, die in allen Stadien der Krankheit wirkt (CAVALCANTE; PEREIRA CASTRO, 2017).

\section{ZIEL}

Präsentieren Sie die Anzahl der gemeldeten Fälle von angeborener Syphilis im Norden Brasiliens zwischen 2014 und 2019 und charakterisieren Sie das epidemiologische Profil der Fälle. 


\section{METHODE}

Eine deskriptive, querschnittliche und retrospektive quantitative Studie wurde anhand von Daten des Notifiable Diseases Information System (SINAN/SUS) der Informatikabteilung des Unified Health System (DATASUS) durchgeführt. Die nationalen Daten wurden nach folgenden Schritten erhoben: Der http://indicadoressifilis.aids.gov.br Link wurde aufgerufen und in "Datenabdeckung" wurden "Regionale und nationale Daten" und in "Unterkategorie" "Brasilien" ausgewählt. Die Schaltfläche "Daten herunterladen" wurde angeklickt und Daten zur angeborenen Syphilis Brasilien entfernt. Anschließend wurde in "Data Coverage" "State Data" ausgewählt und in "Subcategory" wählten wir den Namen jedes Bundesstaates der nördlichen Region Brasiliens, die Tabellen jedes Staates wurden heruntergeladen und die Informationen zur angeborenen Syphilis wurden entfernt. Die Daten wurden in der Excel-Anwendung,einer Komponente der Microsoft Corporation Office-Suite, zusammengestellt.

\section{ERGEBNISSE}

Der Prozentsatz der Fälle von angeborener Syphilis nach endgültiger Diagnose pro Diagnosejahr in Brasilien zwischen 2014 und 2019 ist in Tabelle 1 dargestellt. Der Prozentsatz der Fälle stieg von 12,28\% im Jahr 2014 auf 18,21\% im Jahr 2019. 
Tabelle 1 - Zeigt den Prozentsatz der Fälle von angeborener Syphilis nach endgültiger Diagnose pro Diagnosejahr im Norden Brasiliens zwischen 2014 und 2019.

\begin{tabular}{rrc}
\hline ANO & No CASOS & $\%$ \\
\hline $\mathbf{2 0 1 4}$ & 16353 & $12.28 \%$ \\
$\mathbf{2 0 1 5}$ & 19712 & $14.80 \%$ \\
$\mathbf{2 0 1 6}$ & 21330 & $16.01 \%$ \\
$\mathbf{2 0 1 7}$ & 25037 & $18.79 \%$ \\
$\mathbf{2 0 1 8}$ & 26531 & $19.92 \%$ \\
$\mathbf{2 0 1 9}$ & 24253 & $18.21 \%$ \\
\hline
\end{tabular}

Im Norden Brasiliens betrug der Prozentsatz der Fälle von angeborener Syphilis nach endgültiger Diagnose pro Diagnosejahr zwischen 2014 und 2019 11.071. In diesem Zeitraum stieg die Zahl der Fälle von 11,13\% im Jahr 2014 auf 20,19\% im Jahr 2019 (Abbildung 1). 
Abbildung 1 - Zeigt den Prozentsatz der Fälle von angeborener Syphilis nach endgültiger Diagnose pro Diagnosejahr im Norden Brasiliens zwischen 2014 und 2019.

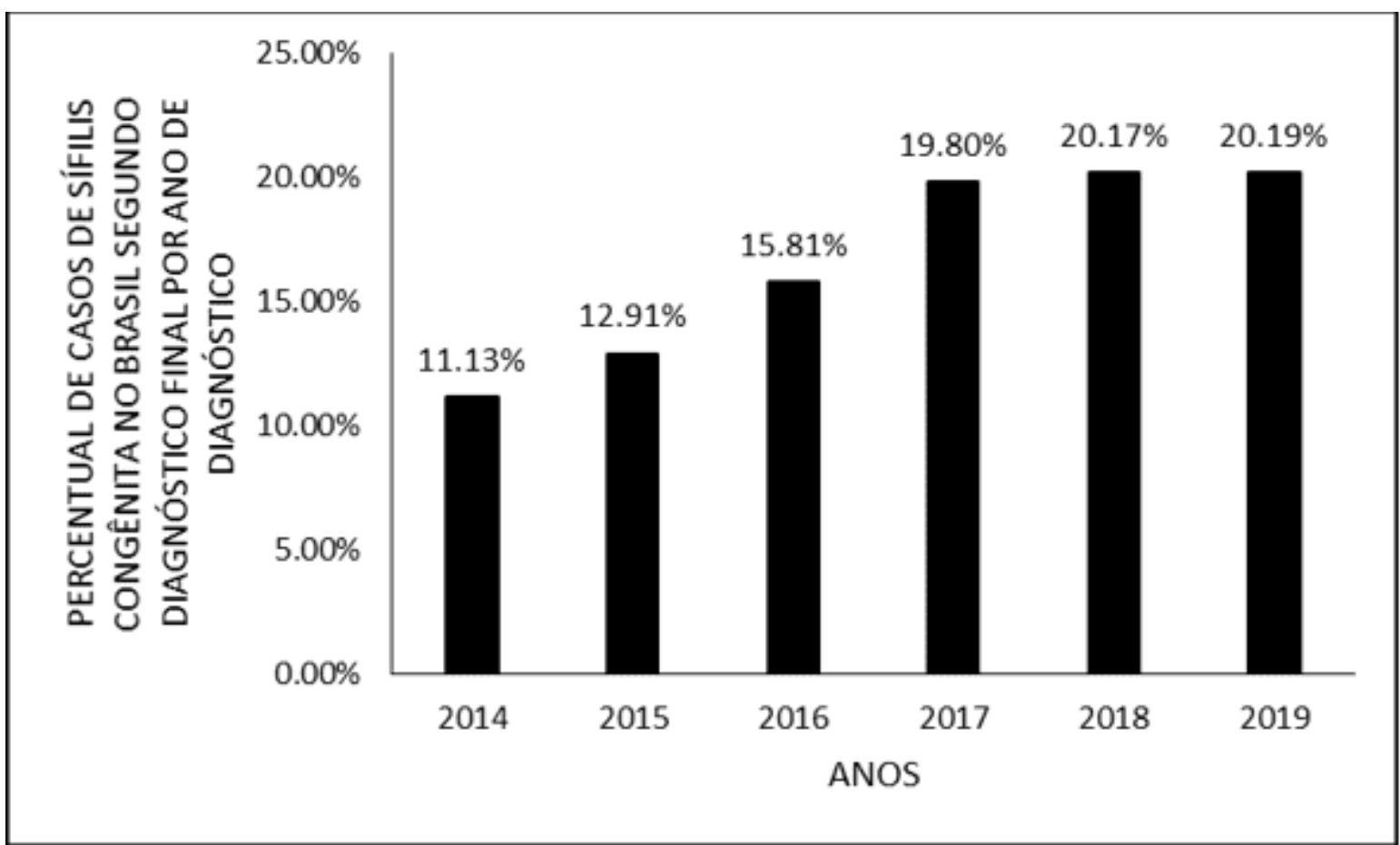

Unter den Staaten in der Region Nord stach Pará mit der höchsten Anzahl von Fällen $(42,08 \%)$ und der Staat mit der niedrigsten Anzahl, der Bundesstaat Roraima (1,83\%) hervor. (Abbildung 2). 
Abbildung 2 - Zeigt den Prozentsatz der Fälle von angeborener Syphilis nach endgültiger Diagnose pro Diagnosejahr, nach Staat in der Nordregion Brasiliens, zwischen 2014 und 2019.

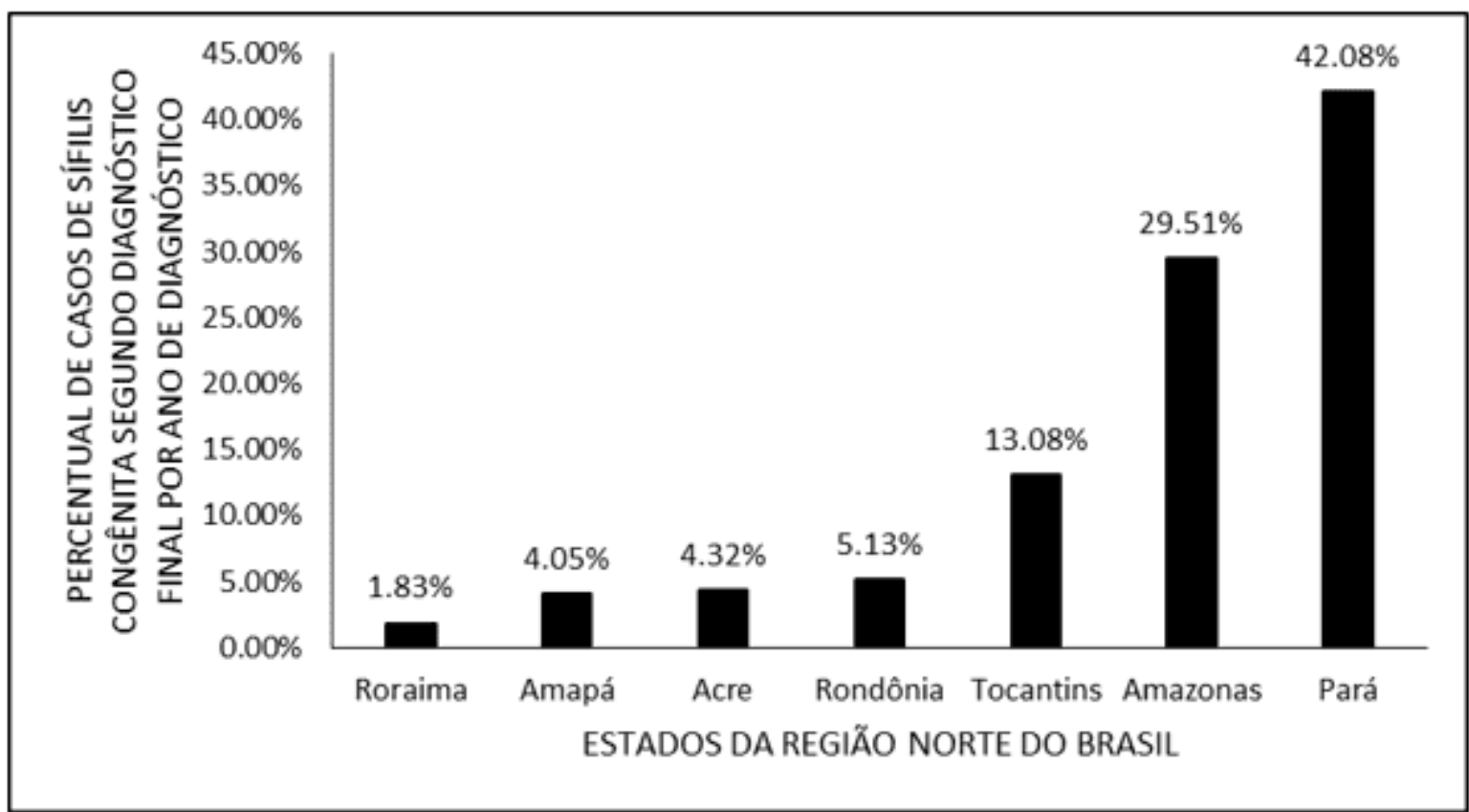

Abbildung 3 zeigt die prozentuale Verteilung der Fälle von angeborener Syphilis in der Nordregion nach zweiter Rasse/Farbe (ethnizität) zwischen 2014 und 2019. Die Daten zeigen eine höhere Anzahl von Fällen in der braunen ethnischen Gruppe mit 9.638 (87,06\%), gefolgt von weißen mit 608 (5,49\%). Die ethnischen Gruppen mit der niedrigsten Zahl waren indigen mit $85(0,77 \%)$ bzw. schwarzen mit 32 (0,29\%). 
Abbildung 3 - Zeigt die prozentuale Verteilung der Fälle von angeborener Syphilis in der Nordregion nach zweiter Rasse/ Farbe (ethnische Zugehörigkeit) zwischen den Jahren 2014 und 2019.

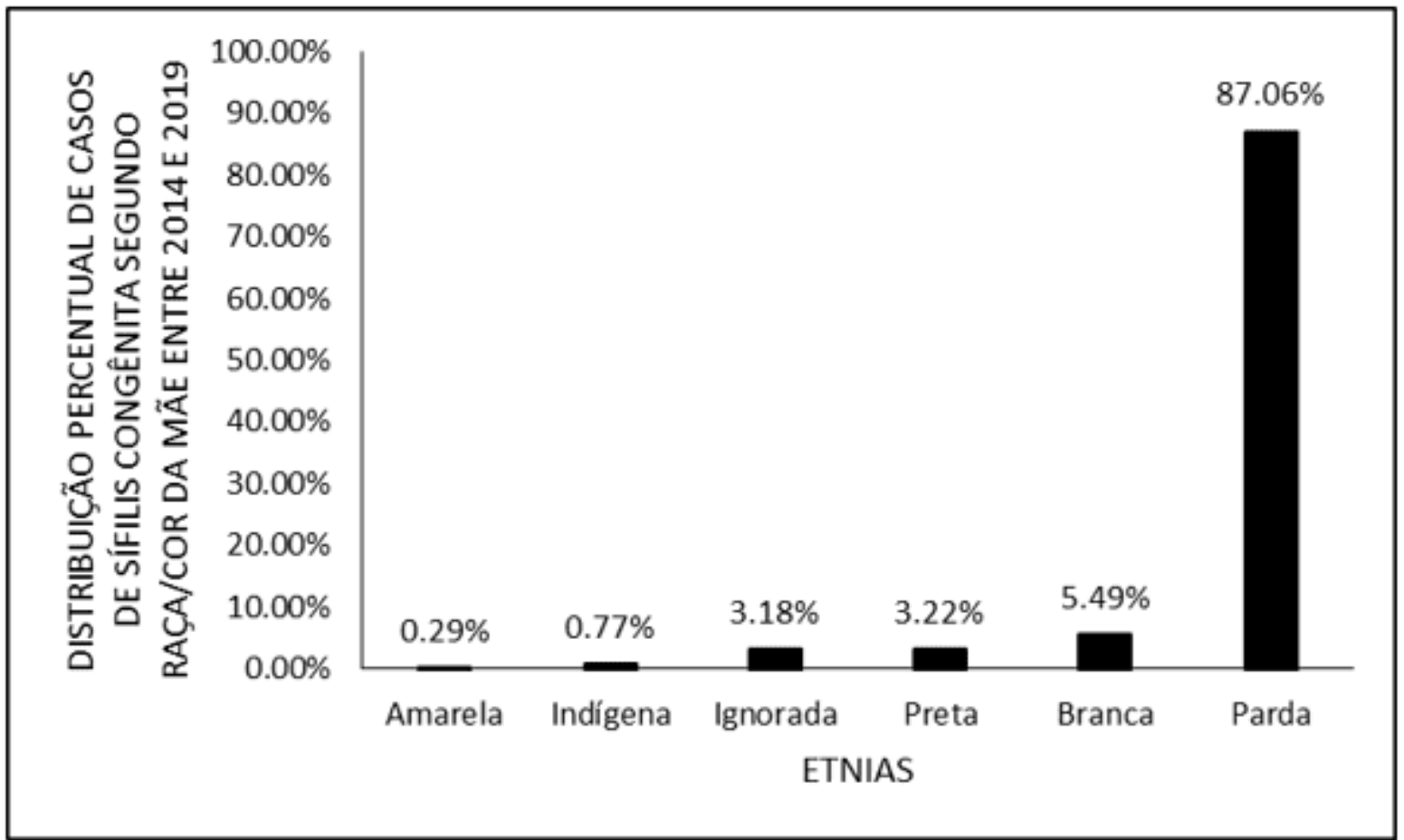

Abbildung 4 zeigt die Anzahl der bestätigten Fälle von angeborener Syphilis in der Region Nord durch pränatale Untersuchung zwischen 2014 und 2019. Die Daten zeigen, dass eine größere Anzahl von Frauen, die sich einer schwangerschaftsvorsorgenden Behandlung unterzogen haben, 8.712 (78,69\%) als diejenigen, die sich nicht 2.048 (18,50\%) unterzogen haben, und diejenigen, die als ignoriert gelten, insgesamt $311(2,81 \%)$ betrugen. 
Abbildung 4 - zeigt die prozentuale Verteilung der Fälle von angeborener Syphilis nach Informationen zur Schwangerschaftsvorsorge der Mutter pro Diagnosejahr zwischen 2014 und 2019.

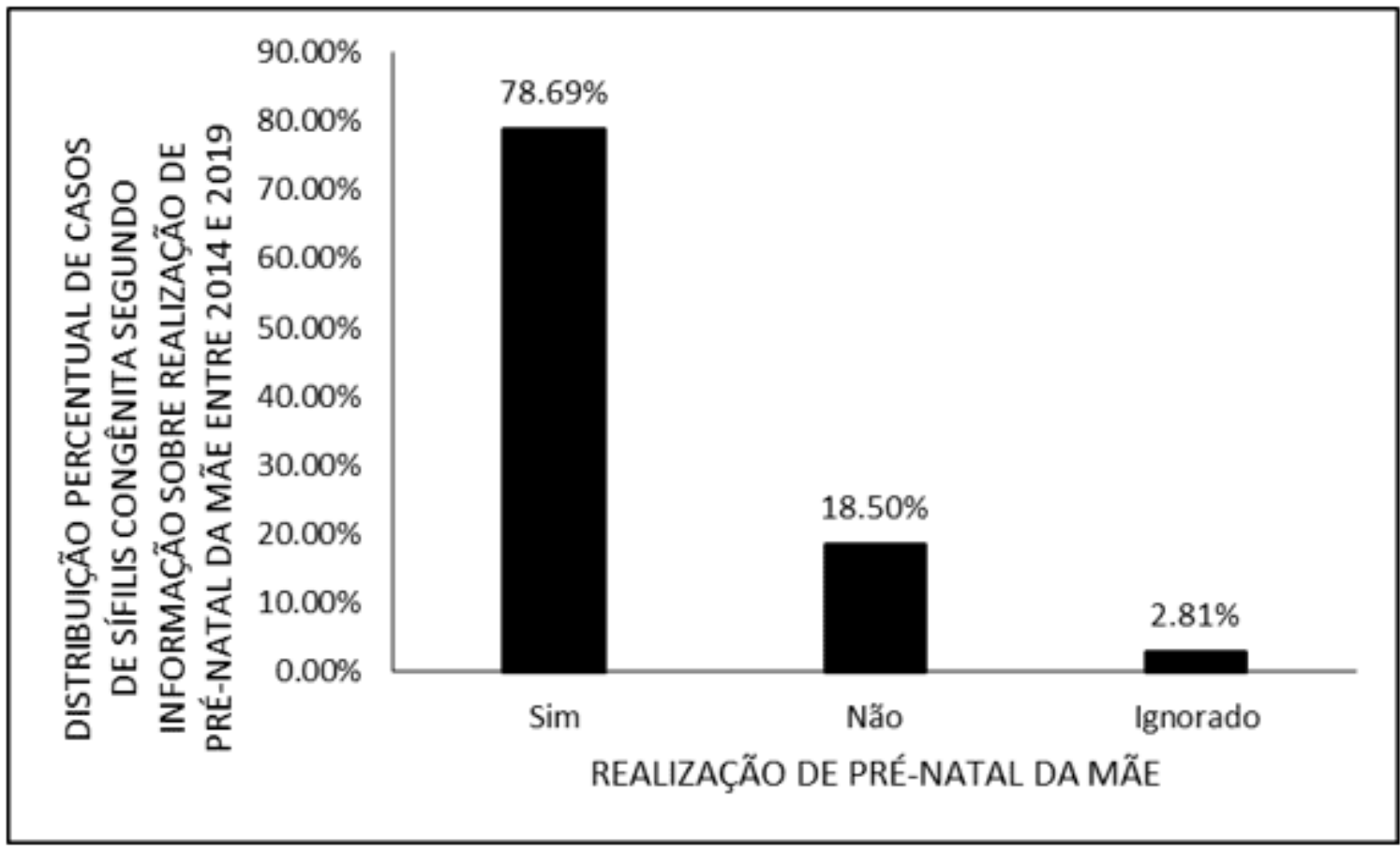

Abbildung 5 zeigt die prozentuale Verteilung der Fälle von angeborener Syphilis in der Region Nord, entsprechend der Schulbildung der Mutter pro Diagnosejahr zwischen 2014 und 2019. Die Daten zeigen eine wichtige Anzahl von analphabetischen Müttern von 62 Frauen $(0,90 \%)$, die Mehrheit der Fälle mit der mütterlichen Schulnote der 5 . bis 8. unvollständigen Grundschule mit 2.362 (33,80\%), wobei eine Sammlung von Frauen die Grundschule abgeschlossen hat von 936 (13,40\%) und ein Teil die High School abgeschlossen hat 1.320 (18,90\%), die niedrigste Anzahl von Müttern mit abgeschlossener Hochschulausbildung betrug 62 (0,90\%) und die Anzahl der ignorierten Mütter betrug 2.243 (32,10\%). 
Abbildung 5 - Zeigt die prozentuale Verteilung der Fälle von angeborener Syphilis in der Region Nord, entsprechend der Schulbildung der Mutter pro Diagnosejahr zwischen 2014 und 2019.

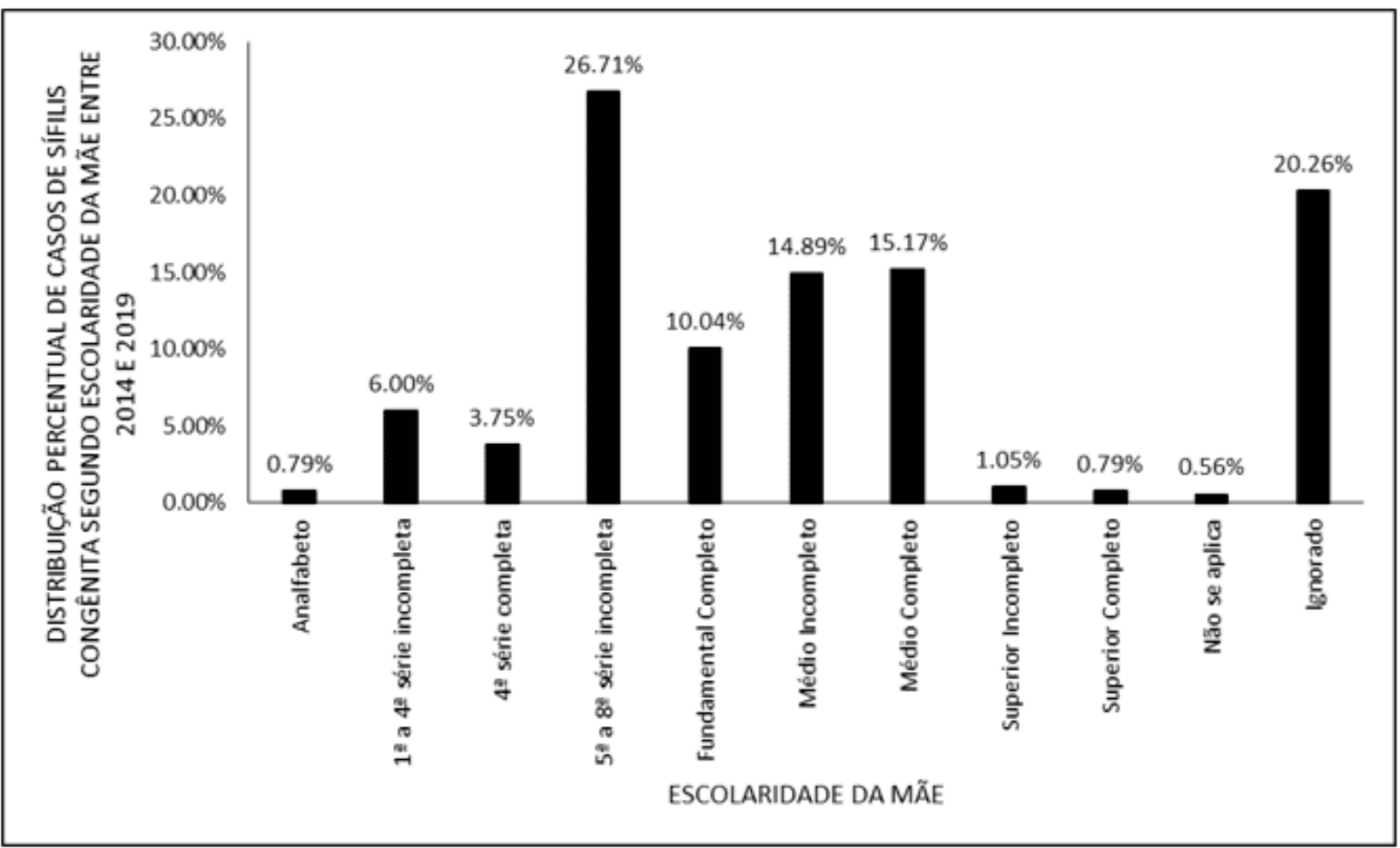

Abbildung 6 zeigt die Anzahl der bestätigten Fälle von angeborener Syphilis in der Region Nord zum Zeitpunkt der mütterlichen Diagnose für Syphilis zwischen 2014 und 2019. Es gibt eine größere Anzahl von Infektionen während der pränatalen Untersuchung mit einer Gesamtzahl von Fällen von 4.862 (43,92\%) und auch zum Zeitpunkt der Entbindung mit 4.258 (39,46\%), wobei eine Minderheit der Fälle in der postpartalen Periode diagnostiziert wurde, mit insgesamt $1.388(12,54 \%)$ und die ignorierten Fälle insgesamt 447 (4,04\%). 
Abbildung 6 - Zeigt die prozentuale Verteilung der bestätigten Fälle von angeborener Syphilis nach dem Zeitpunkt der Diagnose in der Region Nord zwischen den Jahren 2014 und 2019.

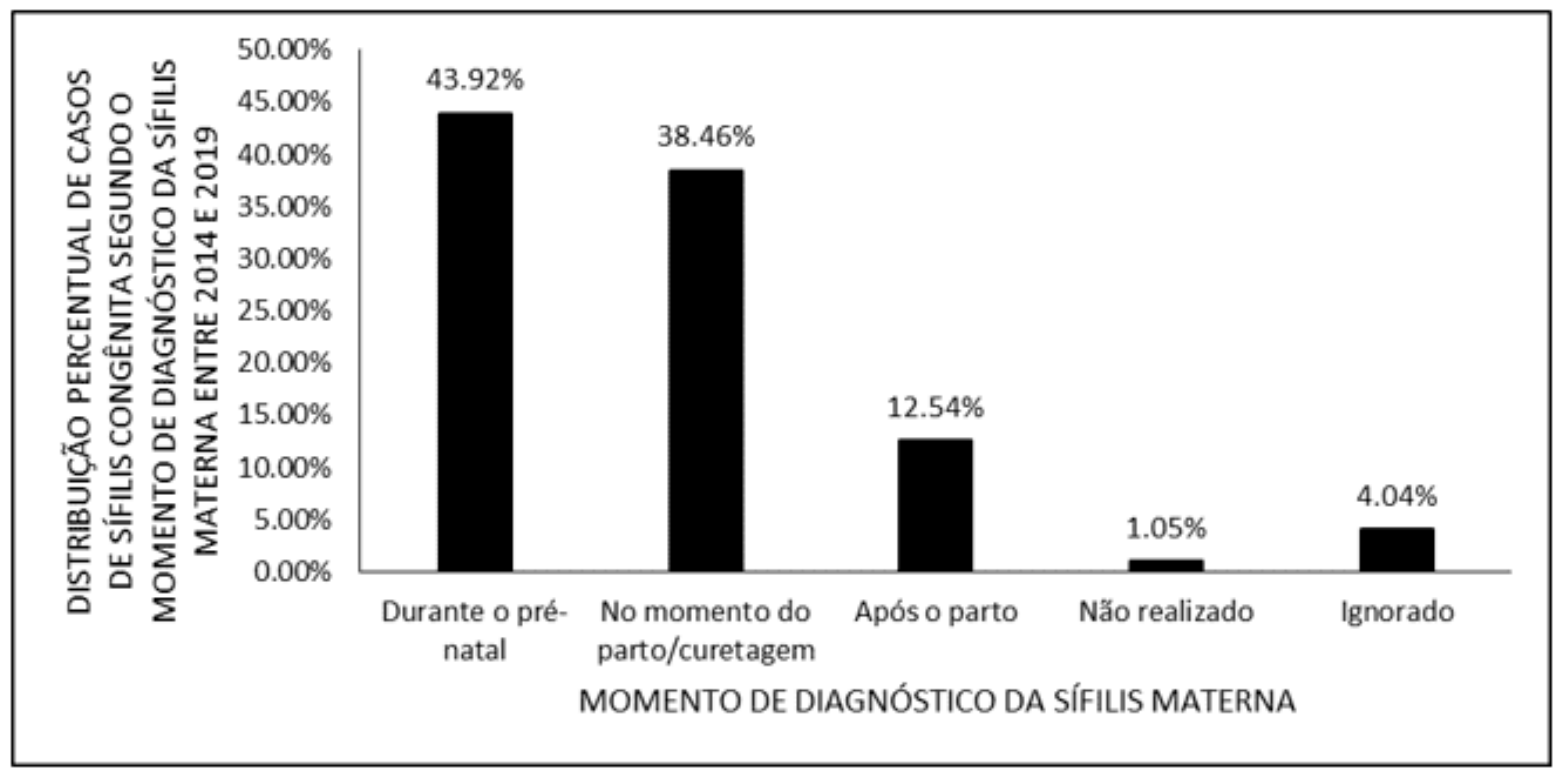

In Bezug auf die variable endgültige Klassifizierung der angeborenen Syphilis wurden die gefundenen Daten 10.475 (94,62\%) als neuere angeborene Syphilis eingestuft; 24 als späte angeborene Syphilis eingestuft (0,22\%); 256 (2,31\%) Abtreibungen aufgrund von Syphilis; und $316(2,85 \%)$ totgeboren durch Syphilis (Tabelle 2). 
Tabelle 2 - Zeigt die prozentuale Verteilung der Fälle von angeborener Syphilis nach endgültiger Diagnose pro Diagnosejahr in der nördlichen Region Brasiliens zwischen 2014 und 2019.

\begin{tabular}{c|c|c}
\cline { 2 - 3 } & $\begin{array}{c}\text { No CASOS } \\
\text { (2014 a 2019) }\end{array}$ & $\%$ \\
\hline $\begin{array}{c}\text { Sífilis congênita } \\
\text { recente }\end{array}$ & 10475 & $94.62 \%$ \\
Sífilis congênita tardia & 24 & $0.22 \%$ \\
Aborto por sífilis & 256 & $2.31 \%$ \\
Natimorto por sífilis & 316 & $2.85 \%$ \\
\hline
\end{tabular}

\section{DISKUSSION}

Von 2014 bis 2019 stieg die Zahl der Fälle (Abbildung 1), insbesondere der Bundesstaat Amazonas, der die Meldezahlen der Krankheit im beobachtungszeitraum um 416\% (Abbildung 2) erhöhte und sogar der Staat der nördlichen Region mit dem höchsten Pro-Kopf-BIP war (IBGE, 2016). Diese Analyse des Zusammenhangs zwischen der Inzidenz der Krankheit und der wirtschaftlichen Kapazität des Standorts wird durch die Tatsache durchgeführt, dass die angeborene Syphilis eine relativ einfache diagnostische Erkrankung ist und völlig vermeidbar ist, wenn die Behandlung von schwangeren Frauen und dem Partner angemessen durchgeführt wird (ARAUJO et al., 2006), die ihre Prävalenz im Allgemeinen mit einer mangelhaften Wirtschaftskraft in Verbindung bringt, um eine qualitativ hochwertige Grundgesundheit zu bieten, genauer gesagt, um eine wirksame pränatale Versorgung für die Bevölkerung anzubieten (ARAUJO et al., 2006).

Eine Unterentwicklung würde jedoch nicht allein die Ursache dafür erklären, dass Amazonas der Hauptanbauzustand der Krankheit im beobachteten Zeitraum ist, denn im Vergleich zu anderen Staaten in der nördlichen Region ist es das beste BIP, so dass die Verbesserung der Meldung der Krankheit im Staat die Tatsache erklären RC: 96427

Zugangslink: https://www.nucleodoconhecimento.com.br/gesundheit/nordbrasilien 
könnte, dass die nördliche Region eine hohe Prävalenz dieser Komorbidität hat. Im Jahr 2016 startete das Gesundheitsministerium von Amazonas eine Dezentralisierungskampagne für Schnelltests auf sexuell übertragbare Krankheiten, die zuvor in FMT (Heitor Vieira Dourado Tropical Medicine Foundation) überkonzentriert war, und verteilte sie an die vier SAEs (Specialized Care Services) der Stadt (BRASIL, 2016).

Noch zu den Perspektiven, die versuchen, die hohe Inzidenz von angeborener Syphilis in der Nordregion zu erklären, hängt die Unterentwicklung auch mit einer Bevölkerung mit einem geringeren Bewusstseinsgrad zusammen, das heißt, es würde nicht nur die Verfügbarkeit von Schwangerschaftsvorsorge ausreichen, sondern das Verständnis der Gesellschaft über die Bedeutung ihrer Realisierung, was hilft zu verstehen, warum es sich um ein Problem der öffentlichen Gesundheit handelt, das in der nördlichen Region Brasiliens immer noch so präsent ist. die zweite brasilianische Region mit dem niedrigsten Pro-Kopf-BIP, die nach dem Nordosten an zweiter Stelle steht (IBGE, 2016).

In Bezug auf die variable Ethnizität in den registrierten Fällen von angeborener Syphilis (Abbildung 3) waren mehr als 90\% der Fälle von gemischter Ethnizität (Rasse), die auch die häufigste ethnische Gruppe in der nördlichen Region ist und $66,88 \%$ der Bevölkerung dieser Region Brasiliens ausmacht (IBGE, 2010).

Es ist auch wichtig, eine Parallele zu zwei in der Forschung gesammelten Daten zu ziehen, der Prozentsatz der infizierten Mütter, die sich einer Schwangerschaftsvorsorge unterzogen haben, beträgt im beobachteten Zeitraum (2014-2019) mehr als 70\% (Abbildung 4), dh die meisten Frauen in der Region machen diese Nachbeobachtung, was eine geringere Inzidenz der Krankheit implizieren sollte. Auf der anderen Seite ist die Anzahl der unbehandelten Partner hoch. Syphilis ist eine Krankheit der sexuellen Übertragung, bei der die Behandlung des Partners obligatorisch ist. Dies spiegelt eine schwierige Realität Brasiliens bei der Durchführung einer effektiven Schwangerschaftsvorsorge wider, das Fehlen einer zufriedenstellenden Nachsorge des Partners in Konsultationen. Eines der Hauptprobleme, das immer noch bei der Kontrolle von sexuell übertragbaren RC: 96427

Zugangslink: https://www.nucleodoconhecimento.com.br/gesundheit/nordbrasilien 
Infektionen (STIs) während der Schwangerschaft besteht, ist der Ansatz des Partners (DUARTE, 2007). Darüber hinaus treten die Probleme mit der Benachrichtigung auf und setzen sich fort, um sie von der Notwendigkeit der Diagnose und Behandlung zu überzeugen (DUARTE, 2007).

Ein weiterer Faktor ist der Grad des Analphabetismus, nur 0,9\% waren Analphabeten und der höchste Prozentsatz ist von Menschen, die mindestens die Grundschule abgeschlossen haben (Abbildung 5). Etwa 8\% der Bevölkerung im Norden Brasiliens sind jedoch Analphabeten, die zweitschlechteste Region, die nach der Nordostregion an zweiter Stelle steht (IBGE, 2019). Dieser Vergleich der Daten führt zu Fragen über die Unterberichterstattung, ob der höchste Prozentsatz von Frauen mit Syphilis in der Region tatsächlich eine höhere Ausbildung hat oder ob der Prozentsatz, der Pflege sucht und die gemeldeten Fälle hat, die Mehrheit ist und derjenige mit der niedrigsten Ausbildung folgt, ohne Pflege zu suchen und die Krankheit nicht gemeldet hat.

Unzureichende Schwangerschaftsvorsorge verhindert Routine für diagnose und wirksame und frühzeitige Behandlung (ARAUJO et al., 2006). Daten zum Zeitpunkt der Diagnose unterstreichen die Bedeutung einer wirksamen Schwangerschaftsvorsorge zur Bekämpfung der Inzidenz von angeborener Syphilis, da die Mehrheit die Diagnose bei der Entbindung oder zum Zeitpunkt der Kürettage hatte (Abbildung 6).

\section{SCHLUSSFOLGERUNG}

Aufgrund der hohen Inzidenz von Fällen von angeborener Syphilis gibt es die Prominenz der Nordregion zum Nachteil anderer brasilianischer Regionen. Die Unterentwicklung der Region ist ein grundlegender Faktor, um diese Tatsache zu erklären, wenn es ein Defizit im öffentlichen Sektor gibt, insbesondere in Bildung und Gesundheit, spiegelt sich die Evidenz in der Bevölkerung wider. In Bezug auf die angeborene Syphilis hatten die meisten schwangeren Frauen angesichts dieser Daten ein niedriges Bildungsniveau, da es an Informationen über die Bedeutung der Schwangerschaftsvorsorge mangelte, sowohl aufgrund des mangelnden Interesses der Bevölkerung als auch der geringen Verbreitung des öffentlichen RC: 96427

Zugangslink: https://www.nucleodoconhecimento.com.br/gesundheit/nordbrasilien 
Gesundheitssystems. Zum Beispiel ist der Bundesstaat Amazonas, der der Staat mit der höchsten prozentualen Rate der im analysierten Zeitraum gemeldeten Fälle war, aufgrund einer höheren Leistung beim Screening von sexuell übertragbaren Infektionen, jedoch ist die größere Verfügbarkeit von Screening-Methoden nicht gleichbedeutend mit einer Verbesserung der Inzidenz von Fällen von angeborener Syphilis.

Darüber hinaus hat die Anzahl der unbehandelten Partner eine große Relevanz, da der häufigste Übertragungsweg der Syphilis der sexuelle Weg ist. Es gibt einen Mangel an Informationen und Widerstand von den meisten von ihnen. Daher ist es notwendig, dass die Schwangere zusammen mit ihrem Partner während der Schwangerschaftsvorsorge gescreent wird, um eine mögliche Reinfektion einer behandelten Patientin oder eine frühzeitige Diagnose zu vermeiden.

Daher wird beobachtet, dass für die Verringerung der Inzidenz von Fällen von angeborener Syphilis eine Reihe von Faktoren erforderlich sind, von der größeren Verfügbarkeit von Informationen über die Bedeutung der Schwangerschaftsvorsorge und ihre Kontinuität durch schwangere Frauen und ihre Partner. Decken Sie daher eine frühzeitige Diagnose und Behandlung ab, um Komplikationen für den Fötus oder neugeborene Menschen zu vermeiden.

\section{VERWEISE}

ARAUJO, Eliete da Cunha; Costa, Kelly de Souza Gama; SOUZA E SILVA, Rafaela; AZEVEDO, Valéria Nascimento; Lima, Fábio André Souto. Importância do pré-natal na prevenção da Sífilis Congênita. Revista Paraense de Medicina, Belém, v. 20, n. 1, p. 47-51, mar. $2006 . \quad$ Disponível em: $\quad$ http://scielo.iec.gov.br/scielo.php?script=sci arttext\&pid=S010159072006000100008\&lng=pt\&nrm=iso Acesso em: 24 maio 2019.

BARROS, Yara Lorrane Souza de. Et al. Numbers of confirmed syphilis cases in pregnant women in Brazil between 2009 and 2013. Revista Científica Multidisciplinar Núcleo do Conhecimento. Year 05, Ed. 11, Vol. 25, pp. 53-61. 
November

2020.

ISSN:2448-0959,

Access

link

in:

https://www.nucleodoconhecimento.com.br/health/syphilis-cases,

DOI:

10.32749/nucleodoconhecimento.com.br/health/syphilis-cases

BRASIL, Ministério da Saúde. Departamento de Doenças de Condições Crônicas e Infecções Sexualmente Transmissíveis: Manaus recebe apoio da ONG AHF para descentralizar 0 atendimento de HIV/aids. 2016. Disponível em: http://www.aids.gov.br/pt br/noticias/manaus-recebe-apoio-da-ong-ahf-paradescentralizar-o-atendimento-de-hivaids _ Acesso em: 23 maio 2019.

BRASIL, Ministério da Saúde. Departamento de Vigilância, Prevenção e Controle das Infecções Sexualmente Transmissíveis, do HIV/Aids e das Hepatites Virais: Protocolo Clínico e Diretrizes Terapêuticas para Atenção Integral às Pessoas com Infecções Sexualmente Transmissíveis (IST). 2018. Disponível em: http://conitec.gov.br/images/Artigos Publicacoes/Diretrizes/PCDT Atencao Integral IST 22-10-18.pdf Acesso em: 23 maio 2019.

CARDOSO, Ana Rita Paulo; ARAÚJO, Maria Alix Leite; CAVALCANTE, Maria do Socorro; FROTA, Mirna Albuquerque; DE MELO, Simone Paes. Análise dos casos de sífilis gestacional e congênita nos anos de 2008 a 2010 em Fortaleza, Ceará, Brasil. Ciência \& Saúde Coletiva. v. 23, n. 2, p. 563-574, 2018. Disponível em: https://www.scielosp.org/article/csc/2018.v23n2/563-574/ Acesso em: 24 maio 2019.

CAVALCANTE, Ana Egliny S; SILVA, Maria Adelane M; RODRIGUES, Antonia Regynara M; NETTO, José Jeová Mourão; MOREIRA, Andréa CA; GOYANNA, Natália F. Diagnóstico e tratamento da sífilis: uma investigação com mulheres assistidas na atenção básica em Sobral, Ceará. DST Jornal Brasileiro de Doenças Sexuais. v. 24, n. 4, p. 245-245, 2012. Disponível em: http://www.dst.uff.br/revista244-2012/4-Diagnostico\%20e\%20Tratamento\%20da \%20Sifilis.pdf Acesso em: 23 maio 2019

CAVALCANTE, Patricia Alves de Mendonça; PEREIRA, Ruth Bernardes de Lima; CASTRO, Jose Gerley Diaz. Sífilis gestacional e congênita em Palmas, Tocantins, 
2007-2014. Epidemiologia e Serviços de Saúde. v. 26, n. 2, p. 255-264, abr./jun, 2017. Disponível em: https://www.scielosp.org/article/ress/2017.v26n2/255264/pt/ Acesso em: 23 maio 2019.

CRT. Centro de Referência e Treinamento DST/AIDS-SP. Coordenadoria de Controle de Doença, Secretaria de Estado da Saúde de São Paulo (SES-SP). Guia de bolso para o manejo de sífilis em gestante e sífilis congênita. 2 ed., 112 p. São Paulo: Secretaria de Estado da Saúde. 2016. Disponível em: http://www.saude.campinas.sp.gov.br/saude/doencas/sifilis/guiadebolsodasifilis_2edi cao2016.pdf Acesso em: 23 maio 2019.

DUARTE, Geraldo. Extensão da Assistência Pré-natal ao Parceiro como Estratégia de Aumento da adesão ao Pré-natal e Redução da Transmissão Vertical de Infecções. Revista Brasileira de Ginecologia e Obstetrícia. v. 29, n. 4, p. 171-174, 2007.

GUINSBURG, Ruth; SANTOS, Amélia Miyashiro Nunes dos Santos. Critérios diagnósticos e tratamento da sífilis congênita. Documento científico Departamento de Neonatologia. São Paulo: Sociedade Brasileira de Pediatria. 2010. Disponível

em: https://www.sbp.com.br/fileadmin/user upload/2015/02/tratamento sifilis.pdf Acesso em: 24 maio 2019.

Instituto Brasileiro de Geografia e Estatística (IBGE). Contas regionais do Brasil: 2010-2014, Coordenação de Contas Nacionais. - Rio de Janeiro: IBGE, 2016. 97 p. (Contas nacionais, ISSN 1415-9813; n. 53). Disponível em: https://biblioteca.ibge.gov.br/visualizacao/livros/liv98881.pdf Acesso em: 24 maio 2019.

Instituto Brasileiro de Geografia e Estatística (IBGE), Diretoria de Pesquisas, Coordenação de Trabalho e Rendimento, Pesquisa Nacional por Amostra de Domicílios Contínua 2016-2018. 2019. Disponível em: https://biblioteca.ibge.gov.br/visualizacao/livros/liv101657_informativo.pdf Acesso em: 24 maio 2019. 
IBGE. Instituto Brasileiro de Geografia e Estatística. Sistema IBGE de Recuperação Automática - SIDRA. $2010 . \quad$ Disponível em: https://sidra.ibge.gov.br/Tabela/3175\#resultado Acesso em: 24 maio 2019.

MOTTA, Isabella almeida; DELFINO, Isabella Rey de Souza; DOS SANTOS, Leticia Vettorazzi; MORITA, Maura Omori; GOMES, Rayanne Gonçalves Dantas; MARTINS, Talita Pouzas Soares; CARELLOS, Ericka Vianna Machado; ROMANELLI, Roberta Maia de Castro. Sífilis congênita: por que sua prevalência continua tão alta?. Revista Médica de Minas Gerais, Belo Horizonte, v. 28. Suppl. 6, 2018. Disponível em: http://rmmg.org/artigo/detalhes/2418 Acesso em: 23 maio 2019.

ROCHA, Karina Dias. Et al. Number of congenital syphilis cases in Brazil between 2009 and Revista Científica Multidisciplinar Núcleo do Conhecimento. Year 05, Ed. 05, Vol. 01, pp. 131-143. May 2020. ISSN:2448-0959 DOI: 10.32749/nucleodoconhecimento.com.br/health/congenital-syphilis-cases

Eingereicht: Dezember 2020.

Genehmigt: Dezember 2020. 